\title{
PSYCHOL: A computer language for experimentation'
}

ROBERT S. MCLEAN, ${ }^{2}$ CARNEGIE-MELLON UNIVERSITY, Pittsburgh, Pennsylvania 15213

Psychologists employing digital computers in the laboratory to control experimental procedure must construct programs. A language is described that incorporates many data representations and manipulations useful in presenting stimuli, collecting responses, and deciding the course of an experiment. PSYCHOL is an extension to a subset of $A L G O L$, with data types extended to include simple lists, strings of text, historic variables, and responses. Timing mechanisms, stimulus presentation, and data recording instructions expand the operations available within the language.

Small digital computers are being used increasingly in the psychological laboratory to control the presentation of stimuli and to collect responses. As laboratory computers become commonplace, more researchers will need to know machine characteristics, ways in which they may be used, and how to program the experiments the researcher wishes to run.

Several discussions now exist of the characteristics of small computers suitable for use in the laboratory, the most comprehensive of use to psychologists being that by Uttal (1968). The concepts have also been discussed by others (Green, 1963; Miller, Bregman, \& Norman, 1965; Cooperband, 1966; Spinrad, 1967).

As was true in the development of general-purpose computers, the advancement of programming techniques and other tools have lagged behind the development of hardware and interfacing technology. The typical small computer for control purposes is supplied with a relatively low-level assembly language and, perhaps, a version of an algebraic language that is suited to numeric computation. But it is rare that a language devoted exclusively to process control is supplied with the programming support. This is due to several factors. First, the range of equipment that might be used in a laboratory suggests some difficulty in devising universal ways of referring to it. Second, the size of the market has been such as to discourage such attempts by computer manufacturers. Finally, psychologists lack a widely used or accepted standard process notation for the description of psychological experiments. The last is a particularly formidable problem. If there were an "algebra" in common use that had already formalized and abstracted the operations inherent in experimentation and combined these with a notation and operation rules there would be little problem in transforming these into a computer language for control of experiments. Witness, for example, the relative ease (at least in retrospect) with which algebraic notation was transformed into FORTRAN and ALGOL. It may be argued that these languages were the result of intense effort by many people and that similar resources are not now being applied to the problem of control computation in psychology. This is all the more reason to suggest that the absence of widely accepted notation is more of a hindrance.

The SCAT system (Grason-Stadler, 1968), which accompanies a particular interface marketed by a manufacturer of psychological apparatus, and the Automatic Contingency Translater (Millenson, 1968) are languages based on a Markovian state-change principle. To date, these have found principal use in animal conditioning experiments and have thus not required as great a richness of data structures and stimulus capability as might be found in some laboratories conducting experiments with human Ss.
A second approach, used at a number of installations, consists of deciding upon a set of operations desired of the system and framing these as parameterized subroutines or the equivalent. These programs tend to be oriented toward a special application and have not found their way into the general literature.

This paper describes a language for specifying experiments that would be applicable to a range of experiments and based on a recognized computer language framework. The language is called PSYCHOL and was implemented on a Honeywell DDP-116 computer at Carnegie-Mellon University; an implementation for the Digital Equipment Corporation PDP-9 is currently under way.

\section{IMPLEMENTATION}

The first implementation of PSYCHOL was accomplished on the computer that is used for control of experiments in the Carnegie-Mellon laboratory. The machine is word-oriented (16 bits/word) with 16K words of core (Honeywell DDP-116). Peripheral storage consists of a disc. The system was implemented to carry on an interactive line-by-line compilation of the program, ignoring an entire line when an error was detected. A front-end file system was added to help preserve the input program text from one test run to the next. Binary versions of the compiled program were also stored on disc.

PSYCHOL compiles the program into an assembler language representation that is temporarily stored on disc. Following completion of the compilation, this assembly language program is processed by an assembler system into the actual machine language program. In the present implementation of the language for the PDP-9, it is anticipated that PSYCHOL will produce an intermediate representation that is similar to assembly language but a step above it so that an additional processor can be used to convert it to an actual assembly language, thereby making it applicable to more than one machine. The current processor is being written in a more universal language, FORTRAN, to further facilitate machine transferability. Also possible is the use of a larger general-purpose machine to compile the program and produce the object version for use in the small laboratory machine.

\section{A PSYCHOL EXAMPLE}

Before examining the features of the PSYCHOL language in detail, it may be useful to consider an experiment and its program. Suppose that $E$ wishes to measure the reaction time of a $S$ to a visual display. A PSYCHOL program for such an experiment is given in Fig. 1. The line numbers are not part of the program, but help in relating the program to the description to follow.

The $S$ responds to the display in the experimental room by pressing a switch that will be called "button" (Line 2). The basic unit of the experiment is a trial, and the number of them in a "block" will be determined by the E just before the experiment is run, by entering a parameter (4). At the beginning of a block of trials, the display scope will present the words "new block" for a period of $5 \mathrm{sec}$ (6). Each trial begins with the display of the word "ready"; after a random interval of 500 to $1,500 \mathrm{msec}$, the word "go" appears (10). This is the signal for the $S$ to respond; doing so causes the scope screen first to become blank (12), and then to display the latency of his response (14). In addition, on the 10th and succeeding trials the average latency of response over the previous 10 trials will be calculated and displayed (17-22). If 10 trials have not yet elapsed, the $S$ sees "average not calculated, $\mathrm{n}=$ " and the trial number. The $\mathrm{E}$ is provided with a record of the 


\begin{tabular}{|c|c|}
\hline 1 & BEGIN \\
\hline 2 & RESPONSE button FROM room; \\
\hline 3 & EVENT block (TIME,NUMBER,ALWAYS); \\
\hline 4 & PARAMETER n.in.block; \\
\hline 5 & HISTORIC last.latency ( 10 ON r); \\
\hline 6 & block: $\mathrm{n}:=$ lat $:=0$; DISPLAY 'new block'; \\
\hline 7 & block + 5 SECONDS: trial: BLANK; CURSOR 50,200; SIZE 2; \\
\hline 8 & DISPLAY 'ready'; \\
\hline 9 & CURSOR 100,60; SIZE 4; \\
\hline 10 & trial + RANDOM $(1000)+500$ MSEC: DISPLAY 'GO'; \\
\hline 11 & PERMIT button; AWAIT button; \\
\hline 12 & BLANK; SIZE 1; CURSOR 1,150 \\
\hline 13 & last.latency $:=$ LATENCY OF button; $\mathrm{n}:=\mathrm{n}+1$ \\
\hline 14 & DISPLAY 'latency', LATENCY OF button; \\
\hline 15 & CURSOR 1,100 \\
\hline 16 & IF $\mathrm{n}>9$ THEN BEGIN \\
\hline 17 & $\mathrm{i}:=-10 ;$ lat $:=0$ \\
\hline 18 & loop: lat $:=$ lat $+\mathrm{i}$ TH OF last.latency; \\
\hline 19 & $\mathrm{i}:=\mathrm{i}+1$ \\
\hline 20 & IF $\mathrm{i}<0$ THEN GO TO loop; \\
\hline 21 & lat $:=$ lat $/ 10$ \\
\hline 22 & DISPLAY 'average for 10 trials =', lat; \\
\hline 23 & END ELSE DISPLAY 'average not calculated, $n=$ ', $n$; \\
\hline 24 & RECORD n, LATENCY OF button, lat; \\
\hline 25 & WAIT $15 *$ LATENCY OF button; \\
\hline 26 & IF $n>n$.in.block THEN GO TO block ELSE GO TO trial; \\
\hline 27 & END; \\
\hline
\end{tabular}

Fig. 1. A sample PSYCHOL program.

trial number, the latency on that trial, and the average of the last 10 latencies (24). An intertrial interval is proportional to the latency of the response (25), after which another trial commences if there are trials remaining in the block (26).

Persons who have used an algebraic language (FORTRAN, ALGOL, or PL/I) will recognize the structure of the arithmetic statements, declarations (2-5), and labels ("block" in 6; "trial" in 7 ). The reserved identifiers (which are shown in all capital letters for clarity, although typographical features have no significance in PSYCHOL) often suggest their functions. The operator ":=" should be regarded as a unitary symbol, an assignment operator performing the function of the equals sign in FORTRAN; the equals sign resumes its function as an indicator of relational equality in the IF statement.

The reserved identifiers used in the example may be classed according to general function: RESPONSE (2) defines the characteristics of the responses that are then processed by PERMIT and AWAIT (11), and that provide the basis for the calculations implied by LATENCY OF $(13,14,24)$. The stimuli are displayed on a scope at a position given by a $\operatorname{CURSOR}(7,12)$ statement in a size of type indicated by SIZE. Displays are removed by BLANK $(7,12)$ and caused by DISPLAY $(6,14)$, the material displayed being indicated following the word DISPLAY. Data is recorded as specified by a RECORD (24) statement or according to an EVENT (3) declaration that causes automatic logging of certain information when the program passes a specified label. A PARAMETER (4) declaration signifies that the values of specified variables are to be supplied at run time. The HISTORIC (5) declaration establishes an automatic means of preserving past values of variables at particular points of the program for later use by the program (as in the computation of the average over 10 trials in Lines 18 to 20). For mnemonic ease, an identifier may be interspersed with periods-as in "n.in.block" (4) and "last.latency" (5)-which are ignored by the processor.

With this glimpse of a program, we now proceed to a detailed catalogue of PSYCHOL's features.

\section{THE PSYCHOL LANGUAGE}

The following is a description of the major features of the PSYCHOL language; a specification in a more formal notation can be found in McLean (1968). Where examples are given in the following, the reserved identifiers (words with special meaning) are shown in capitals; lower-case letters denote variables supplied by the user.

PSYCHOL is built upon a subset of ALGOL (Naur, 1963). ALGOL was selected because it offers a useful structure on which to build special-purpose program structures. For instance, the basic unit of an ALGOL program is the statement, which is a unit of program that performs a computation, e.g., assigning a value to a variable. A statement may, however, be a more complex 
combination of other statements, inserted between the word brackets, BEGIN and END. In this way, a simple logical structure may be elaborated to almost any degree of complexity. The elaboration might be likened to the structure of an outline where any entry may have subsidiary entries but may itself be a subsidiary entry in higher entries.

Knowledge of the ALGOL base language is assumed in the following description, which is limited to a discussion of the extensions to that base. The experimental control features of PSYCHOL are discussed under the topics of data representations, conventions for denoting the passage of time, mechanics of stimulus and response processing, data manipulation, data recording, parametrization of experiments, subroutining, and assembly language escape features.

\section{Data Representations}

Many of the features that make higher-level programming languages useful result from the ways in which data to be manipulated can be organized. Thus, languages having representations for arrays of numeric information are likely to be more appropriate for some purposes than are languages that organize data into list structures of arbitrary symbols. It is likely that psychological research will have use for both numeric arrays and symbolic lists, as well as alphanumeric strings of information. PSYCHOL provides a form of each of these and comparable forms of manipulations. Numeric information may be stored and manipulated in integer variables and one-dimensional arrays in the manner specified by ALGOL. The need to declare all variables has been removed from ALGOL by making the assumption in PSYCHOL that nondefined variables are integer variables. Another implementation might allow the entire range of variables declarable under the specification of ALGOL.

In addition to numeric variables, additional forms of representation that may be declared include lists of integers, alphanumeric strings, lists of strings, lists of tasks, historic variables, and responses.

Lists are of three types: integers, strings, or tasks. A list is an ordered set of integers (or other numeric types in a fuller implementation) that can be initialized when the program is written to contain any numeric values in a specified order. Manipulations are available to permit successive reference to the items in order, to initialize the pointer for such references to the beginning of the list, and to permute randomly the items of the list.

A string list is a list of arbitrarily long strings of text that are associated in an order and are subject to the same manipulations provided for integer lists.

A task list provides an ordering to portions of executable program that may be executed in order, randomized, or otherwise manipulated as are other lists.

Lists are provided primarily to serve as organizers and storage for stimulus material; they are ordered, but may also be randomized. The provision of the three data types within the list capability expands the type of stimuli that may be represented: numbers, strings, and programs. Task lists permit the user to program stimulus generators that may create stimuli or values internal to the program.

Strings provide for the symbolic representation of alphanumeric information as text; their primary use is for stimulus presentation and data recording. A text string may be associated with an identifier by declaration or it may be used in several contexts as a string constant simply by enclosing it within single quotes.

Historic variables are integer variables that are capable of retaining information for a specified interval within the experiment. The variable is associated with a particular label within the program and acts as a push-down variable with respect to that label. Each time control passes that label, the contents of that variable are pushed one step into history and a new storage register is made available for that variable.

The historic variable is implemented as a circular list so that only a finite amount of history is retained; this amount is specified by the user in the declaration. List manipulations take account of this mechanism and provide ways in which previous values of the variable may be accessed. When using nonlist manipulations of the variable, only the current value is referenced, so that the variable appears to act just as any other single variable.

Event declarations are used to record the general progress of the experiment. The user is able to specify points in the program where a notation is to be made in the output, providing a trace of the order in which events occurred within the experiment. Optional specifications may be included in the event declaration to cause the time or sequential numbering of the event to be recorded with the notation. Similarly, one can specify that repeated occurrences of an event not be recorded unless different events intervene. This may turn out to be a useful tool for debugging a program as well as data recording.

Response declarations will be mentioned below.

\section{Timing}

The times at which interactions occur between $S$ and the computer are usually of interest to the researcher and must be controlled. Relative timing within the program is made available by automatically associating with each label in the program the time at which control last passed that point. Minimum constraints on the time at which events are permitted to occur may then be obtained by referring to a label plus a time increment. This construction may take the form of a "temporal label," in which case it behaves syntactically as if it were a label. In the following example, suppose that control passes a statement labeled L1 at a particular time; should the control attempt to pass the statement labeled L1+100 MSEC before 100 msec have passed, execution of the program will be suspended until that condition has been met. (Elipses "..." represent any intervening part of the program.)

$$
\text { L1: ...; } 1+100 \text { MSEC: ... }
$$

The time expression may be an arithmetic expression, so that the time delay is variable, e.g., it may depend upon previous responses. In addition, there are instructions to allow the program to wait for a period of time or to wait until a period of time after the passing of a label (equivalent to the temporal label):

\section{WAIT 3 SECONDS}

WAIT UNTIL L2 + 2 SECONDS;

A third temporal contruction permits the repeated execution of a statement (or compound statement) until a particular time:

\section{UNTIL L1+400 MSEC DO ... ;}

In addition to controlling the times at which events occur under program control, it is desirable to be able to record and manipulate the time. A parameterless function CLOCK has the value of time since the beginning of the experiment in a convenient time unit (e.g., seconds). The latency of each response is also available and may be used by the program in arithmetic expressions.

\section{STIMULI AND RESPONSES}

The stimuli and responses in a psychological experiment are 
equivalent to the computer's output and input, respectively. While it would be possible to provide the traditional READ and WRITE statements for this purpose, the ways in which the FORMAT might be specified seems to provide an additional complication for the researcher. A somewhat different approach has been taken here, one which may be installation dependent, but which is hopefully easier to use.

Having decided to divorce stimuli and responses from the input/output similarity inherent in most computer languages, the dissimilarities become more obvious. Stimuli are initiated by the computer whereas responses are initiated (if at all) by the $\mathbf{S}$. A program may explicitly progress to the point in time when a stimulus must be presented. It cannot, however, guarantee that a response will be present. A complex stimulus (a line of text, perhaps) may be handled as a single entity and output (apparently) as a single unit by passing it to a monitor or hardware device that can deal with it as such. A complex response (e.g., a word typed by a S) cannot be apprehended by the computer as a single entity until the entire response has been assembled by a program. The format of the input character (or word) stream from $\mathrm{S}$ will vary considerably from one experiment to another or even within the same experiment. The differences lead to separate handling of stimuli and responses.

The representations of stimuli and responses are of two types: Simple stimuli and responses are communicated through a several-bit buffer (typically one computer-word in length), where each bit is likely to have some meaning in the experiment (e.g., an output bit is connected to a light; an input bit is the output of a switch or button). More complex stimuli (e.g., strings) are communicated to devices or service routines that can handle them as a unit (e.g., a display scope).

Responses resemble data in that they form information that should be available for computational manipulation by the program. Because responses are initiated by $S s$ who are performing apart from the program, they occur independently of it. The additional distinguishing features of responses are that they originate in some particular experimental equipment and that they are usually acceptable only at certain times. The equipment of origin contains some information about the conversions that may be necessary to map responses onto internal variables, and so this information must be given in a declaration. The response declaration associates the equipment with a response identifier. The response may be a simple response or a string response, in which case, information concerning the termination characters for the string is also declared. Response declarations are made at the beginning of a program along with the other data declarations. Thereafter, a response is referred to by its identifier, almost as if it were data, and may be manipulated similarly to other data of similar types.

The acceptability of a response is determined by two commands, PERMIT and PROHIBIT, which make the system responsive to S's inputs. Thus, if a response "resp" is to be used, the following might be written (recall that user-provided identifiers for variables, labels, etc., are shown here in lower case):

\section{BEGIN \\ RESPONSE resp FROM experimental.room;}

\footnotetext{
L1: PERMIT resp;<smiles>[IH2]</smiles>

L2: PROHIBIT resp;

...etc....
}

The "experimental.room" conveys information about the kind of data and source of the response; it would be established as a convention local to the particular laboratory. A response made by the $S$ following the execution of the statement labeled $L 1$ and before L2 is reached would be associated with the identifier "resp."

The response identifier may be used in two ways in data manipulations. First, it may be used as a Boolean value, in which case it conveys the value TRUE if a response has occurred since the PERMIT was executed and the value FALSE if the response has not occurred. Second, it may be used as a simple (integer) variable in arithmetic and logical computations, where it can be tested for equality with expected responses. In addition, various other manipulations are available, extending ALGOL to include logical operators.

The LATENCY OF a response may be obtained by simply requesting it; the value is the time in milliseconds since the last PERMIT of that response:

time :=LATENCY OF resp;

This statement assigns to the variable "time" the elapsed time between execution of "PERMIT resp" and the actual occurrence of the response.

If it is desired to suspend execution until such time as the response is made, the construction,

\section{AWAIT resp;}

accomplishes that. It is also possible to set an expiration time on the period one is willing to wait for the response, as in

\section{AWAIT resp FOR 3 SECONDS;}

which automatically continues execution of the program when the response occurs or after $3 \mathrm{sec}$ have elapsed, whichever comes first. The phrase beginning "FOR" is a "temporal modifier," and will be used extensively in defining the temporal scope of stimulus statements.

Stimuli are somewhat simpler and assume the one-word output register mentioned above for the simpler forms. Individual bits in the output register may be set or reset according to the $1 \mathrm{~s}$ in the expression following the respective command:

\section{TURN ON 1; \\ TURN OFF $x+y$;}

These commands respectively turn on the least significant bit of the output register (the binary representation of the value 1) and turn off any bits that correspond to the 1-bits in the sum of $x$ and $y$, leaving other bits unchanged. The latter is included to show that the value may be any arithmetic or logical expression. In addition, several shift operators are also included to aid in the positioning of bits that are to be set or reset. The entire register may be set to the value of an expression or variable, e.g.,

\section{OUTPUT z;}

Any of these stimulus commands may have a temporal modifier that states the time at which the operation will be reversed or, in the case of OUTPUT, all bits will be set to zero:

\section{OUTPUT $\times$ FOR 3 SECONDS;}

This instruction thus sets the output register to the contents of the variable $\mathrm{x}$ for a period of $3 \mathrm{sec}$, and then resets the register to all zeroes. 
More complex stimuli may be displayed on an appropriate device with a set of elementary text display commands. These include a way of specifying an origin of a text string and the size of characters to be used on the scope as well as a DISPLAY command. There is a BLANK instruction to remove the display. Examples are:

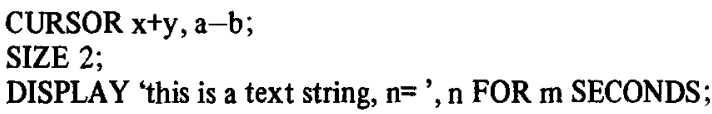

This displays the text between the single quotes, followed by the numerical value of " $n$ " (if it is a numerical variable), in characters of arbitrary size 2, beginning at a point on the display screen having a horizontal coordinate of " $x+y$ " and a vertical coordinate of "a-b." Note that the generalized arithmetic expression may be substituted in any of the places where a single variable may be used. In addition, the text string might have been a string variable or an item from a string list. Where numerical variables, expressions, or values are given, decimal conversion is performed on the value. The optional temporal modifier has been included; the display will be BLANKed automatically after $\mathrm{m}$ seconds have elapsed.

\section{Manipulations of Strings and Lists}

Each list has a pointer associated with it that determines the currently referenced item. Referring to a list by its identifier without other modification causes the current location to be treated as if it were a single variable (as was discussed under historic variables, above). The pointer may be moved along the list or reset to the top of the list by the following:

\section{NEXT OF list.name;}

INITIALIZE list.name;

In addition, if NEXT OF is used as a Boolean (i.e., in an IF-statement), it not only moves the pointer, if possible, but produces the value TRUE or FALSE according to whether or not it was able to do so, i.e., if there are still items on the list to which the pointer may be moved.

List items may be referenced by a special construction:

$$
\text { arithmetic.expression TH OF list.name; }
$$

The "arithmetic.expression" determines which of the items of the list "list.name" is evaluated, according to one of three rules. The Oth item is the current item, as if there were no modifier. Any positive value designates the item in that position of the list, starting from the top of the list as Item 1. Negative numbers are taken circularly backwards from the current pointer item, so that the -1 TH OF a list is the item just above the current item, treating the last item as just before the first item of the list. This is a mechanism that is consistent with the historic variables that are implemented as circular lists. Thus, the declaration,

INTEGER LIST numbers := 1,2,3,4,5,6,7,8,9,0;

and the expression,

\section{TH OF numbers}

would yield the value 4 . The expressions,

0 TH OF numbers

-5 TH OF numbers would depend upon where the current pointer was located. Suppose that it pointed at 7 . The first example would have the value 7 , and the second, 2 . If the pointer was at 3 , the second example would be evaluated 8 .

Strings may be broken apart for stimulus generation and response checking. The language allows the specification of BEFORE, AFTER, and TO with the specification of a string to the left and a character in single quotes to the right. Thus, in a list of paired associates, one might represent the items of interest as one of the following:

\section{item BEFORE '-’ \\ item AFTER '-’ \\ item TO '-}

This would provide the pair up to the hyphen located between the stimulus and response, the response member, and the stimulus member with the hyphen, respectively. Because of the substitutability of constructions within the PSYCHOL structure, the "item" may, of course, be a string expression or an item from a string list.

\section{Data Recording}

Data recording is simplified. In implementing PSYCHOL, it is assumed that a computer will be the primary tool in analysis of the data obtained from the experiment, so that the primary job of the data-recording mechanism is to record values on command in some delimited, machine-readable form. The RECORD statement provides this function; it consists of the word RECORD and a list of items to be recorded, separated by commas. The items may be variables or expressions or constants; they may take on numeric or string values and the PSYCHOL processor supplies appropriate conversions to output readable values for each type of data. The end of a record (line) may be indicated by the reserved identifier "CR," denoting a "carriage return." Example:

\section{RECORD 'The results are', LATENCY OF resp, resp, CR}

This would output the string indicated, along with the values of the latency of the response "resp" and that response itself. The EVENT construction mentioned earlier also performs a data-recording function.

\section{Parametrization}

The experiment may be such that it is desirable to set the values of some variables when the experiment is to be run. This is a way of indicating to the program that a certain experimental condition is to be run with the particular S. At any time in the program, a notation of this fact may be made by associating the variable identifier with the word PARAMETER:

\section{PARAMETER trials, inter.trial.interval;}

This would cause the system to query the user at the beginning of each run concerning the values of the variables trials and inter.trial.interval to be used for this S.

\section{Subroutining}

PSYCHOL provides the ability to write parts of a program separately from the main body of the program and to cause these to be executed at will within the program. These parts of program are called TASKs and are declared in the outermost block head. These are similar to ALGOL procedures, but do not allow parameters. This restriction is compensated partially by the 
ability to create lists of TASKs that may be manipulated as any other list, so that the selection of a task, say, stimulus generation, may be sequenced or randomized easily. The task may be evoked by the construction

or

DO task.name;

\section{DO n TH OF task.list.name;}

This can lead to useful constructions, particularly when one may be generating instances of complex concepts.

\section{Assembly Language Escape}

If PSYCHOL doesn't permit a desired operation, there is the ability to provide the required coding in the assembly language of the computer in question. This feature is not usually desirable, but it is provided for the sake of inclusiveness. The escape feature may be used to code a small portion of a program, relying on the organization of PSYCHOL for the rest of the experimental specification.

\section{LANGUAGE EVALUATION}

The usage of PSYCHOL has not yet been extensive enough to provide data concerning the extent to which the language provides natural ways of expressing the control requirements of a psychological experiment. In lieu of a consensus of informed users, we must resort to a discussion of the ways in which PSYCHOL meets the likely demands of experimentation, or comparison with other languages for similar purposes. One user found the lack of ability to define lists of lists to be a restriction. The language leaves untouched the possibility of providing specifications for automatic generation of stimuli unless they are provided by the user's programming. One might envision a language in which the characteristics of stimuli were specified and the stimuli were generated as needed during the course of the experiment. Additional work might be undertaken to make the language more like the discourse of the psychologist, less like the programming language on which PSYCHOL is built.

Certain functions of PSYCHOL might be more highly automated, reducing the "housekeeping" chores that the writer must do. But the amount of such work already assumed in PSYCHOL is considerable and approaches that which differentiates an algebraic language from an assembly language in numerical computation. Help might be provided in the area of overall decision-making within the program, as an example. The historic variable represents a start at this, but at present decisions must be programmed in detail, not stated as simple conditions, e.g., "if the last $n$ responses were instances of (a particular class of responses) then..." The design of more useful features is founded on two considerations: one is finding ways in which to represent appropriate data for decisions and to manage it efficiently; the other is creating ways in which to formulate decisions that will be useful to the experimental design. Sequential statistics and branching teaching devices are instances of the beginning of the use of techniques that might be applied to the experimental control problem to a greater extent. This is likely to be the major need in the field of experimental control techniques for considerable time to come.

\section{REFERENCES}

COOPERBAND, A. S. The use of a computer in conducting psychological experiments. Behavioral Science, 1966, 11, 207-211.

GRASON-STADLER. The SCAT primer (preliminary). West Concord, Mass.: Grason-Stadler Co., 1968.

GREEN, B. F., JR. Digital computers in research. New York: McGraw-Hill, 1963.

McLEAN, R. S. PSYCHOL: A formal language for the control of psychological experiments. Unpublished doctoral dissertation, Carnegie-Mellon University, 1968.

MILLENSON, J. R. A general language for on-line control of psychological experimentation. DECUS Proceedings. Digital Equipment Corp. Maynard, Mass., Spring 1968.

MILLER, G. A., BREGMAN, A. S., \& NORMAN, D. A. The computer as a general purpose device for the control of psychological experiments. In R. W. Stacy and B. Waxman (Eds.), Computers in biomedical research. Vol. 1. New York: Academic Press, 1965. Pp. 467-490.

NAUR, P. (Ed.) Revised report on the algorithmic language ALGOL 60. Communications of the ACM, 1963, 6, 1-17.

SPINRAD, R. J. Automation in the laboratory. Science, 1967, 158, 55-60. UTTAL, W. R. Real-time computers-technique and applications in the psychological sciences. New York: Harper \& Row, 1968.

\section{NOTES}

1. This paper is based upon a doctoral dissertation at Carnegie-Mellon University. The research was supported by a grant from the Public Health Service, National Institute of Mental Health, Research Grant MH-0772, and by a grant from the Mellon Foundation.

2. Now at The Ontario Institute for Studies in Education, Department of Computer Applications. 